\title{
The Application of Improved Self-adaptive Genetic Algorithm in the Distribution Network Reconfiguration
}

\author{
Siwei Wei ${ }^{1}$ \\ Wuhan University of Technology \\ Wuhan, China, 430070 \\ waosfengw@hotmail.com
}

\author{
Ruoxi Wang ${ }^{2}$ \\ HuaZhong Normal University \\ Wuhan, China, 430079 \\ ruoxiwang@163.com
}

\begin{abstract}
This paper proposes a kind of encoding method with the loop switch number as gene and the number of branches of the systematic loop as the length of the chromosome, greatly shortening the length of the chromosome. And this paper also takes a kind of effective means in genetic operation, which is eliminating island and loop circuit, thus greatly decreasing the generation of infeasible solution in the operation to gene and increasing the searching ability and speed of the algorithm. The result indicates that, the combination of adaptive control and genetic algorithm, the adaptive adjusting to the crossover rate and mutational rate accelerate the searching speed of the algorithm, and effectively avoid immature convergence.
\end{abstract}

Keywords- Self-adaptive; Genetic algorithm; Distribution network; Reconfiguration

\section{INTRODUCTION}

A. Merlin presented the concept of reconfiguration of distribution network with some assumptions which are showing load by means of constant pure active component current source, the voltage angle being ignored, not considering constraint condition of network, calculating network flow by means of DC power flow. He confirmed a kind of network structure having the minimum network losing, by using Heuristic Rules, Branch and Delimitation strategy. The shortcomings of this method are that the restraints of network have not been considered, and the use of a great deal of DC power flow calculations is a waste of time.

Tim Taylor used heuristic best-first search strategy as far as possible to eliminate the combination of the switch states of violating the constraint condition, in order to reduce the state space, which is asked for searching ${ }^{[1]}$.

S. Civanlar deduced a formula of the change of network losing when a group of load transfers from one feeder to another, and pointed out that only when load transfers from one point of low electromotive force to another of high electromotive force and the potential difference between these two points is large enough, it is probable to cause the decrease of network losing ${ }^{[2]}$. Darish Shrimohammad is as target function according to the minimum power loss. This method firstly calculates the initial power flow in radiation net, in order to contribute the optional flow mode, closes all the switches, and forms few-mesh distribution system of having some loops; and secondly, it calculates the power flow, gets each value to inject to the current; at the time it solves the optional flow, and then unlocks a loop according to the minimum branch of the current, up to change radiation net ${ }^{[3]}$

This paper uses improved self-adaptive genetic algorithm, comprehensively considers the specialties of distribution network and adaptive genetic algorithm, and proposes a kind of encoding method with the loop switch number as gene and the number of branches of the systematic loop as the length of chromosome, greatly shortening the length of the chromosome. In genetic operation, this paper takes a kind of effective means, which are eliminating island and loop circuit, thus greatly decreasing the generation of infeasible solution in the operation to gene, increasing the searching ability and speed of the algorithm. The combination of adaptive control and genetic algorithm, and the adaptive adjusting to the crossover rate and mutational rate as well, accelerate the searching speed of the algorithm, and effectively avoid immature convergence.

\section{A MATHEMATICAL MODEL}

\section{A. Considering the least time of switching manipulation}

In order to prolong working life of the switches and offer convenience to operators to manipulate, the minimality of the times of operating switches as the objective function is used in the reconfiguration of distribution network ${ }^{[4]}$.

$$
F_{k}=\sum_{i=1}^{m 1}\left(1-y_{i}\right)+\sum_{j=1}^{n 1} z_{j}
$$

In the expression above, $y_{i}$ and $Z_{j}$ denote respectively the states of the section switches and the interconnection switches after the reconfiguration, being 1 when closed, and being 0 when disconnected; $\mathrm{m} 1 、 \mathrm{n} 1$ is respectively the number of the section switches and the interconnection switches in distribution network.

B. Considering network losing to be minimum ${ }^{[4]}$

$$
P_{\text {loss }}=\sum_{i \in F C} r_{i} \frac{P_{i}^{2}+Q_{i}^{2}}{U_{i}}
$$

In the expression above,

$r_{i}$ is the impedance on the feeder;

$P_{i}$ is the active power of flowing through in feeder $\mathrm{i}$;

$Q_{i}$ is the reactive power of flowing through in feeder i;

$U_{i}$ is the voltage on the feeder $\mathrm{i}$;

FC is the set of all closing feeders in network.

Combining literature $[5,6]$, this paper comprehensively 
considers the income of decreasing network losing and the cost of switch operation, and is able to make the distribution network have the maximum income during some time $\mathrm{T}$, and obtain the multi-objective function of the reconfiguration of distribution network.

$$
F=\max \left[k_{1} \times T \times\left(P_{\text {loss }}-P_{\text {loss } 1}\right)-k_{2} \times F_{k}\right]
$$

In the expression above, $k_{1}$ is unit price, $T$ is the time interval of reconfiguration; $P_{\text {lossl }}$ and $P_{\text {loss }}$ are respectively the network losing fore and after reconfiguration; $k_{2}$ is the cost of the switch to open and close the operation for one time; $F_{k}$ is the number of times of switching manipulation when reconfiguration for one time.

\section{Constraint conditions}

1) The constraint of power flow $\sum_{i=1}^{n} S_{i j}=D_{j}$

2) The constraint of circuit capacity $S_{i} \leq S_{i \max }$

3) The constraint of node voltage $V_{i \min } \leq V_{i} \leq V_{i \max }$

4) radialized network constraint having to maintain the radialization of distribution network, and being unable to appear looped network.

In the expression above, $S_{i}$ and $S_{\text {imax }}$ are respectively the calculated value and the maximum permissible value of the power of flowing past of each circuit; $V_{\text {imin }}$ and $V_{\text {imax }}$ are respectively the value of lower and upper limit of node voltage.

\section{SELF-ADAPTIVE GENETIC ALGORITHMS}

Genetic algorithms(GA) is one kind of the most main methods, firstly presented by Prof. Holland in Michigan University, US in 1960s, the basic thought of which is survival of the fittest. In this algorithm, the choice of crossover rate $\mathrm{Pc}$ and mutational rate $\mathrm{Pm}$ is the key to influence the action and the function of GA, and influence directly the convergence of the algorithm, which is the large $\mathrm{Pc}$ is, the faster the speed of creating new individual is. However, the possibility that genetic mode is broken is larger if Pc is over-large; the search progress will be very slow if Pc is over-small. With regard to the mutational rate $\mathrm{Pm}$, if $\mathrm{Pm}$ is over-small, it is uneasy to create new individual structure; nevertheless, the algorithm will change the pure random search and cannot reflect the specialty of GA that is hard to generate new gene, causing the algorithm to be precocity. Standard genetic algorithm usually chooses these two parameters according to experience and repetition test to confirm, and the values are fixed. In order to solve this problem, Srinivas etc. presented self-adaptive genetic algorithm, making Pc and Pm change by themselves along with the change of fitness ${ }^{[7]}$. The main idea is that, when the fitness of each individual in population tends to be uniform or local optimum, Pc and Pm increases, while the fitness of group relatively disperses, Pc and Pm decreases. Self-adaptive genetic algorithm guarantees global convergence property, as well as decreases the iterations, increasing the convergence rate greatly.

\section{THE APPLICATION OF IMPROVED SELF-ADAPTIVE GENETIC ALGORITHM IN RECONFIGURATION OF DISTRIBUTION NETWORK}

\section{A. Encoding scheme}

In the whole distribution network, compared to normally close ordinary switches, interconnection switch is the relative small part, and when a random interconnection switch gets closed, it is inevitable to generate looped network in distribution network. In order to maintain distribution network working arborescently, the section switch in corresponding looped network is disconnected. Meanwhile, the switch that is connected with the power source should be the location of switching on, and moreover, the switch that is not in isolated branch of the loop should also be the location of switching on (if not, it will lead to the island). Therefore, these switches could be deleted when the chromosome is encoding, as well as meaning that the switches that consist of the chromosome are just the ones, which locate intra-loop and the ones, which don't locate intra-loop, don't connect with the power source, and don't be isolated in the branch. Of course, the switch set consisting of the chromosome cannot exist in the repetitive switch. According to the specialty, this paper structures a chromosome according to effective switch states as chromosome contents.

The representative system of three feeders and nodes is shown as Fig. 1

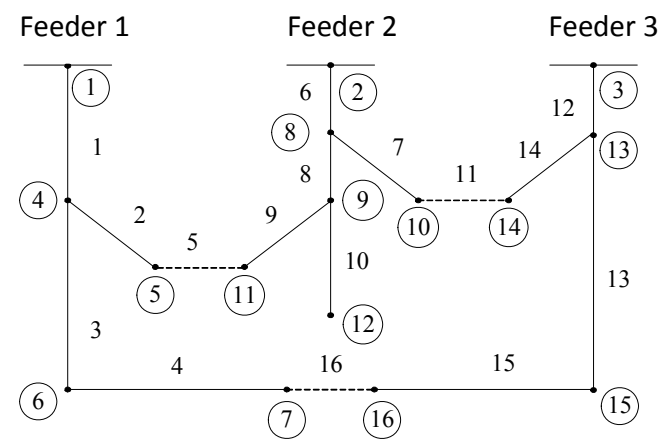

Fig. 1 The representative system of three feeders and nodes

As is indicated in Fig. 1, the state of the branch 10 between 9 and 12 must maintain closed, and thus it could erase its corresponding place in the chromosome, and may not influence the optimizing result. However, the load of node 12 must add to node 9 . In addition, branch 1, 6 and 12 should maintain closed, so the length of chromosome is shortened from 16 to 12 , which increases the rate of feasible solution in searching volume.

It would generate a series of looped network, closing interconnection switch 5,11, and 16, choosing three looped networks, whose branch is the least as basic candidate unit. As is shown in Fig.1, the switch connection loop network 1 contains is branch 1-2-5-9-8-6; the switch connection loop network 2 contains is branch 6-7-11-14-12; the switch connection loop network 3 contains is branch 1-3-4-16-15-13-12. After deleting branch 1, 6, 10, 12, and repetitive nodes, the surplus branch connection, which all 
the loop networks contain according to nodes' serial number to seriate are ranked from small to large. The ranking result is $2,3,4,5,7,8,9,11,13,14,15,16$, which constitutes chromosome by the states of these switches. The basic constitution principle of chromosome is respectively to choose one branch switch to constitute an initial chromosome, such as $5|14| 4$, compiling chromosome is 110011111011.

\section{B. Initial population}

Firstly, generate a chromosome which's each place is all 1 , then disconnect randomly a certain switch, which participates in encoding in a certain loop network, the reason for which is that the switches in the loop are all operating ones, meaning that the corresponding place in the chromosome is 0 . Meanwhile, disconnect the corresponding places in all loop networks that contain this switch. After that, repeat the previous operations in surplus loops, until all the loops are opened, at that time, the chromosome corresponds an individual. In a similar way, generate chromosome whose number is the same as population scale, thus the initial population is obtained.

\section{Adaptive value of chromosome}

Adaptive value is the accordance of genetic algorithm guiding the direction of search. Firstly, adaptive value should be guaranteed to be positive, and secondly optimizing direction of target function must correspond to the increased direction of adaptive value. The target function of network reconfiguration receptively considers the line loss and the time of switch minimum, belonging to the question of the optimization of the minimum, and thus the target function need to be transformed to get fitness function. This paper uses the following form.

$$
\text { Max } F i t(f(x))=1 / f
$$

\section{Improvement of genetic operation}

1) Improvement of choosing: In the process of genetic operation, this paper combines fitness proportion selection method and optimal individual retention method, by firstly using fitness proportion selection method to select, via cross-matched generating the young generation, after that using optimal individual retention method to copy older generational optimal individuals according to $10 \%$. Meanwhile, in order not to change the population number, the worst individuals need to be weed out $10 \%$ from the new population, meaning that the fitness value of these individuals is the least. The proposed method inherits the advantages of fitness proportion selection method and optimal individual retention method. It can increase competing pressure suitably, and reflect the law of survival of the fittest in nature, as well as avoid the individuals of high-fitness being eliminated, and decrease the possibility that the search sink into local optimum because of improper selection.

2) Improvement of the operation of crossover and variation: Because the fitness average value of population is able to reflect the quality of whole population preferably, when the fitness average value of the two individuals which participating in crossover is less than the one of the population, and moreover when the fitness average value of the two individuals which participating in crossover is more than the one of the population, one-point cross is used.

Crossover rate and mutational rate do adaptive adjustment according to the expression as follow.

$$
\begin{gathered}
P_{c}=\left\{\begin{array}{cc}
P_{c}-\frac{\left(P_{c 1}-P_{c 2}\right)\left(f^{\prime}-f_{a v g}\right)}{f_{\max }-f_{a v g}}, & f^{\prime} \geq f_{a v g} \\
P_{c 1}, & f^{\prime}<f_{a v g}
\end{array}\right. \\
P_{m}=\left\{\begin{array}{cc}
P_{m}-\frac{\left(P_{m 1}-P_{m 2}\right)\left(f_{\max }-f_{a v g}\right)}{f_{\max }-f_{a v g}}, & f \geq f_{a v g} \\
P_{m 1}, & f<f_{a v g}
\end{array}\right.
\end{gathered}
$$

In the expression above, $f_{\max }$ is the maximum adaptive value in population; $f_{\text {avg }}$ is the average adaptive value in the population of every generation; $f$ ' is the adaptive value of the biggish one in two individuals which need cross; $f$ is the adaptive value of individuals which need for variation.

As long as setting the values of $P_{c 1}, P_{c 2}, P_{m 1}, P_{m 2}$ (taking values in $[0,1]), P_{c}$ and $P_{m}$ can adjust by self-adaption. This paper does trial calculation in accordance to examples, in operation taking suitably $P_{c l}=0.9, P_{c 2}=0.6, P_{m l}=0.01$, $P_{m 2}=0.001$.

\section{E. Stopping criterion of the algorithm}

This paper uses the method which combines the least maintaining generations of optimal individual and the maximum genetic generations as stopping criterion. When the optimal individuals have no changes in specified generations, it is considered to have already attained the optimal solution, having the optimal individual in current group as optimal solution to the question; otherwise, it stops when attaining the maximum genetic generation, having the optimal individual of the final generation as optimal solution. That specified number of generation is ordinary as a matter of experience to do trial calculation. In this paper, the least maintaining generations of optimal individual defines 20 generations, and the maximum genetic generations defines 300 generations ${ }^{[8]}$.

\section{SimUlation}

This calculating example use American PG\&E 69 nodes distribution system ${ }^{[9]}$, as is shown in Fig. 2, rated voltage is $12.66 \mathrm{kV}$, the whole load is $3802 \mathrm{~kW}+\mathrm{j} 2694 \mathrm{kvar}$. The other parameters are $100 \mathrm{MVA}$ as standard power and $12.66 \mathrm{kV}$ as standard voltage.

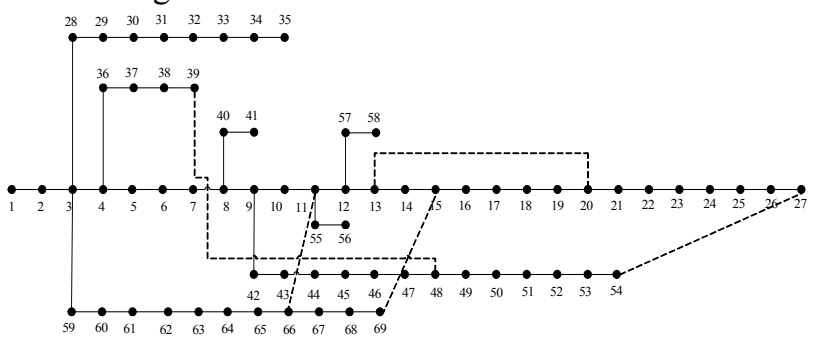

Fig. 2 American PG\&E distribution system with 69 nodes 
To simplify this distribution network, all the switches among line 1-2, 2-3, 3-28, 28-29, 29-30, 30-31, 31-32, 32-33, 33-34, 34-35, 8-40, 40-41, 12-57, 57-58, 11-55 and 55-56 cannot be disconnected, and otherwise there is island to appear. In this example, the length of chromosome is 57 , the population scale is 50 , and the price of electric $k_{l}$ is $0.5 \mathrm{YUAN} / \mathrm{kWh}$, the time interval of reconfiguration $\mathrm{T}$ is 24 hours, the fee that the switch operates 1 time is 7 YUAN. The contrast-calculating result between the method in this paper and vague self-adaptive genetic algorithm in literature [10] is shown as Table 1.

TABLE I Comparison of the original distribution network and reconfiguration

\begin{tabular}{|c|c|c|c|}
\hline & $\begin{array}{c}\text { Before } \\
\text { configuration }\end{array}$ & Literature[10] & $\begin{array}{l}\text { method } \\
\text { in this } \\
\text { paper }\end{array}$ \\
\hline $\begin{array}{l}\text { connection } \\
\text { of opening } \\
\text { switch }\end{array}$ & $\begin{array}{l}11-66 \\
13-20 \\
15-69 \\
27-54 \\
39-48 \\
\end{array}$ & $\begin{array}{l}11-66 \\
13-20 \\
14-15 \\
47-48 \\
27-54 \\
\end{array}$ & $\begin{array}{l}11-66 \\
13-20 \\
14-15 \\
47-48 \\
51-52 \\
\end{array}$ \\
\hline $\begin{array}{l}\text { Voltage in } \\
\text { nadir (p.u.) }\end{array}$ & 0.881737 & 0.926316 & 0.9381 \\
\hline $\begin{array}{c}\text { Network } \\
\text { losing } \\
(\mathrm{kW}) \\
\end{array}$ & 236.8 & 102.47 & 99.7562 \\
\hline $\begin{array}{l}\text { fees of the } \\
\text { total } \\
\text { network } \\
\text { losing } \\
\text { (YUAN) }\end{array}$ & 2841.6 & 1229.64 & 1197.07 \\
\hline $\begin{array}{c}\text { fees of } \\
\text { switch } \\
\text { operation } \\
\text { (YUAN) }\end{array}$ & 0 & 28 & 42 \\
\hline $\begin{array}{l}\text { total } \\
\text { revenue } \\
\text { (YUAN) }\end{array}$ & 0 & 1583.96 & 1602.53 \\
\hline
\end{tabular}

As is presented in table 1, the reconfiguration method in this paper need to open disconnecting switch 14-15, 47-48, and 51-52, and close interconnection switch 15-69, 27-54,
39-48, which operates switches 6 times. Compared with literature [10], the fee of switch operation is more, but using the method proposed in this paper can effectively decrease network losing, increase the quality of voltage, and then when the time interval of reconfiguration $\mathrm{T}$ is 24 hours, the total revenue is higher than the one in literature [10], further confirming the feasibility of this algorithm.

According to the proposed method, continuous operating the optimizer 50 times, the best one is to converge in iteration of the seventh generation, the worst one is to converge in iteration of the twenty-seventh generation, and the most are to converge in iteration of the twentieth generation.

\section{CONCLUSIONS}

This paper consideres comprehensively the specialty of distribution network and self-adaptive genetic algorithm, improves the self-adaptive genetic algorithm, and confirms the effectiveness and rapid convergence of improved self-adaptive genetic algorithm for reconfiguration of distribution network. The result indicates that the used method has good advantages with regard to avoiding sinking into local optimum or approached global optimum, in order to further expand the application of the genetic algorithm in reconfiguration of network distribution.

\section{REFERENCES}

[1] Tim Taylor, David Lubkeman. Implementation of Heuristic Search Strategies for Distribution Feeder Reconfiguration. lEEE Trans on Power Delivery.1990,5(1):239-246

[2] S. Civanlar, J.J. Grainger. Distribution Feeder Reconfiguration Algorithm for Loss Reduction of Distribution Systems. Electrical Power and Energy System,1999,21(9):137 144

[3] Shirmohammadi D, Hong H.W. Reconfiguration of electric distribution networks for resistive line losses reduction. IEEE Trans on Power Delivery.1989,Vo1.4,No.2:pp 1492-1498

[4] Lu Yao-wu. Reconfiguration of Distribution network Based on self-adaptive genetic algorithm [J]. Central China Electric Power, 2005, 18(6), 16-18.

[5] Wang Zhi-yu et al. reconfiguration of distribution network based on time segmented [J]. Relay, 2006, 34(8), 35-39.

[6] Liu Wei, Han Zhen-Xiang. Reconfiguration of distribution network based on time interval. Automation of Electric Power System, 2006, 30(10), 33-38.

[7] Wang Xiao-ping, Cao Li-ming. Genetic algorithm- theory, application, and software implementation [M]. Publishing House of $X i^{\prime}$ an Jiaotong University, Xi'an (in Chinese).

[8] Bi Peng-xiang, Liu Jian et al. improved genetic algorithm in reconfiguration of distribution network [J]. Automation of Electric Power Systems, 2002, 25(1):57-61.

[9] Liu JIan, Bi Peng-Xiang, Dong Hai-Peng. Simplified analysis and optimization to complex distribution network [M]. Beijing. China Electric Power Press, 2002.

[10] Zhang Zhong-Cheng, Wang Chun. Application of vague self-adaptive genetic algorithm in reconfiguration of distribution network [J]. Jiangxi Electric Power, 2006, 30(2): 6-8. 